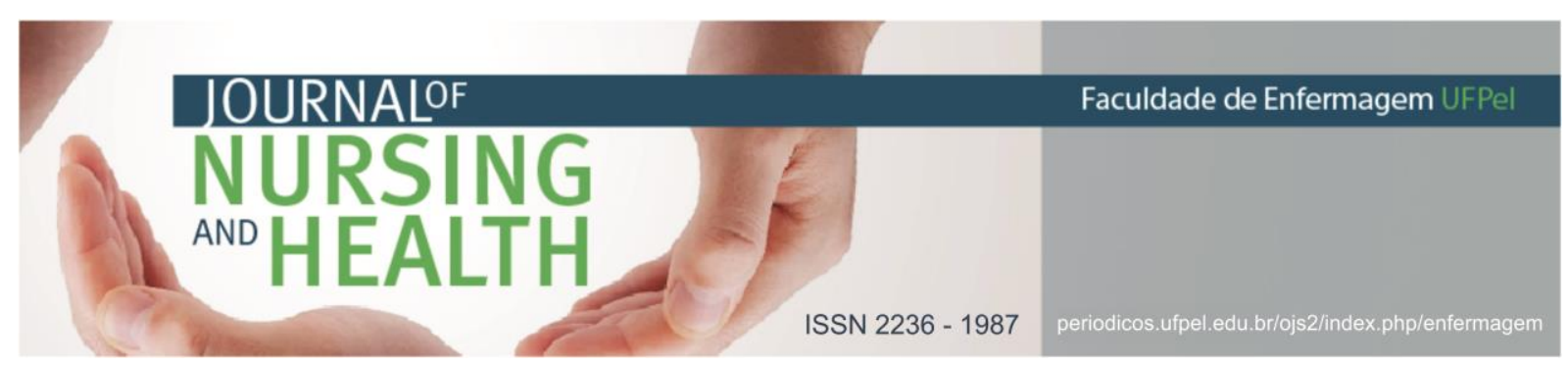

ARTIGO ORIGINAL

\title{
Inventário de ansiedade Traço-Estado de gestantes
}

\author{
State-Trait anxiety inventory of pregnant women
}

\author{
Inventario de ansiedad Rasgo-Estado de las embarazadas
}

Alves, Jheynny Sousa; ${ }^{1}$ Siqueira, Hedi Crecencia Heckler de²; Pereira, Queli Lisiane Castro³

Alves JS, Siqueira HCH, Pereira QLC. Inventário de ansiedade Traço-Estado de gestantes. J nurs health. 2018;8(3):e188307

\section{RESUMO}

Objetivo: identificar o nível de ansiedade e o perfil das gestantes usuárias do Sistema Único de Saúde de Pontal do Araguaia, Mato Grosso. Métodos: pesquisa descritiva e exploratória com abordagem quantitativa a 53 gestantes usuárias do serviço de pré-natal de Pontal do Araguaia, realizou-se a coleta de dados com entrevista semiestruturada, aplicou-se o Inventário de Ansiedade Traço-Estado. Resultados: houve o predomínio de primigestas jovens, pardas, do lar, ou seja, sem emprego formal, casadas, com ensino médio, renda familiar até dois salários mínimos. Não houve correlação positiva entre a situação da gestante no mercado de trabalho e o escore de ansiedade, as casadas possuem estado ansioso menor do que as em união consensual e as gestantes negras possuem estado ansioso elevado. Conclusões: ser casada implica menores níveis ansiogênicos, enquanto ser negra implica maiores níveis ansiogênicos.

Descritores: Ansiedade; Gestação; Saúde da mulher

\begin{abstract}
Objective: to identify the level of anxiety and the profile of pregnant women users of the Unified Health System of Pontal do Araguaia, Mato Grosso. Methods: descriptive and exploratory research with a quantitative approach to 53 pregnant women users of the prenatal service of Pontal do Araguaia. The data collection was performed with semi-structured interview. The State-Trait Anxiety Inventory was applied. Results: there was a predominance of primigravid young, brown, housework at home, that is, without formal employment, married, with high school, family income up to two minimum wages. There was no positive correlation between the situation of the pregnant woman in the labor market and the anxiety score. The married women had an anxious state lower than those in a consensual union and the black pregnant women had an elevated anxious state. Conclusions: being married implies lower anxiety levels while being black implies higher anxiogenic levels.
\end{abstract}

Descriptors: Anxiety; Pregnancy; Women's health

1 Enfermeira. Secretaria Municipal de Saúde de Barra do Garças (MT). Universidade Federal de Mato Grosso (UFMT). E-mail: jheynny_sousa@hotmail.com http://orcid.org/0000-0001-5961-4873

2 Enfermeira. Doutora em Enfermagem. Universidade Federal do Rio Grande (FURG). E-mail: hedihs@gmail.com http: / / orcid.org/0000-0002-9197-5350

3 Enfermeira. Doutora em Ciências. Universidade Federal do Mato Grosso (UFMT). E-mail: quelilisiane@hotmail.com http://orcid.org/0000-0001-6965-4887 


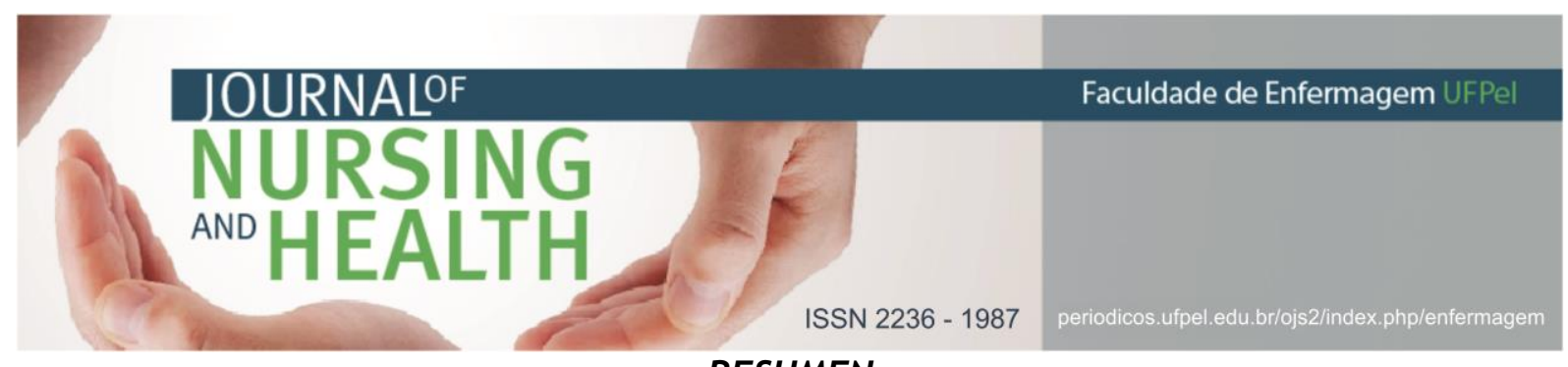

RESUMEN

Objetivo: identificar el nivel de ansiedad y el perfil de las gestantes usuarias del Sistema Único de Salud de Pontal do Araguaia, Mato Grosso. Métodos: investigación descriptiva y exploratoria con abordaje cuantitativo a 53 gestantes usuarias del servicio de prenatal de Pontal do Araguaia, se realizó la recolección de datos con entrevista semiestructurada, se aplicó el Inventario de Ansiedad Rasgo-Estado. Resultados: hubo el predominio de primigestas jóvenes, pardas, dueñas de casa, o sea, sin empleo formal, casadas, con enseñanza media, ingreso familiar hasta dos salarios mínimos. No hubo correlación positiva entre la situación de la gestante en el mercado de trabajo y la puntuación de ansiedad, las casadas poseen un estado ansioso menor que, las en unión consensual y las gestantes negras poseen un estado ansioso elevado. Conclusiones: ser casada implica menores niveles ansiogénicos mientras que ser negra implica mayores niveles ansiogénicos.

Descriptores: Ansiedad; Embarazo; Salud de la Mujer

\section{INTRODUÇÃO}

O ciclo gestacional é um período marcado por inúmeras modificações que necessitam de um processo de adaptação contínuo, no qual se cumprem diversas tarefas de desenvolvimento. A mulher deverá se adaptar tanto às mudanças ocorridas no seu corpo quanto à mudança de papeis gerada pela maternidade, ${ }^{1}$ tal como: de sua condição de filha para a de mãe, além do reajuste do seu relacionamento conjugal, sua situação socioeconômica e profissional, que são mais impactantes em primíparas, porém multíparas podem vivê-las com intensidade. ${ }^{2}$ Esse período de transição é marcado por um aumento na vulnerabilidade emocional e social, ocorrendo incertezas e expectativas diante do nascimento, em que se idealiza um filho perfeito, saudável e sem necessidade de internação.

Tendo em vista um processo que impõe tantas exigências à mulher, é notória a presença da ansiedade, mesmo que excluindo a sua dimensão patológica, os estudos são consensuais quanto à existência de uma morbidade aumentada do tipo ansiosa ou depressiva durante a gravidez. ${ }^{3-6}$
Altos níveis de ansiedade possuem comprovadas implicações na saúde bem-estar da mãe, bem como implicam de forma direta na saúde e desenvolvimento do feto, desde o intra-útero, apontando os riscos de sofrer malformações congênitas, ser prematuro e apresentar baixo peso ao nascer, o que evidencia a interferência direta da ansiedade materna no funcionamento neurocomportamental fetal. ${ }^{4}$ Dessa forma, objetivou-se: identificar o nível de ansiedade e o perfil das gestantes usuárias do Sistema Único de Saúde de Pontal do Araguaia, Mato Grosso (MT).

\section{MATERIAIS E MÉTODOS}

Realizou-se pesquisa descritiva e exploratória com abordagem quantitativa. Participaram 53 gestantes usuárias do serviço de prénatal de Pontal do Araguaia-MT, inseridas no Programa de Humanização no Pré-Natal e Nascimento (PHPN), do Sistema Único de Saúde (SISPRENATAL). Posterior ao parecer 1.842.272, emitido pelo Comitê de Ética em Pesquisa do Campus Universitário do Araguaia pela Universidade Federal do Mato Grosso, 


\section{JOURNALOF \\ NURSING \\ ANO HEALTH \\ ISSN 2236 - 198}

Quanto à raça ou cor da pele das entrevistadas $\mathrm{n}=33 \quad(62 \%)$ se consideram pardas, $n=12(23 \%)$ brancas e $n=8(15 \%)$ negras. No que diz respeito ao estado civil, 21 (40\%) são casadas, $16(30 \%)$ vivem em união consensual, $15(28 \%)$ são solteiras e uma (2\%) é divorciada. Em relação ao grau de escolaridade, $n=25 \quad(47 \%)$ possuíam ensino médio completo, $n=18$ (34\%) o fundamental e $n=10$ (19\%) tinham o superior completo.

Em relação à ocupação, a variável mais significativa observada foi a "do lar", totalizando $n=14$ (26\%), seguida das estudantes $n=11(21 \%)$, professoras $\mathrm{n}=5$ (9\%). As demais ocupações tiveram percentual entre 2 e $4 \%$ e estavam ligadas ao setor terciário da economia. Dentre as gestantes, $n=28$ (53\%) eram economicamente ativas, as demais não tinham rendimentos.

No que tange à renda familiar mensal, $n=24$ (45\%) possuem renda de 1 a 2 salários mínimos ( $R \$ 954,00$ ), $\mathrm{n}=16 \quad(30 \%)$ inferior a um salário mínimo, $n=8(15 \%)$ entre dois e três salários mínimos, $\mathrm{n}=3(6 \%)$ entre três e quatro salários mínimos e $n=2$ (4\%) maior que quatro salários mínimos. Neste estudo, embora os dados tenham demonstraram que as gestantes economicamente inativas possuem a média de Ansiedade-TRAÇO $=49$ (Percentil>75), categorizada como Ansiedade Elevada, enquanto as economicamente ativas, possuem média de Ansiedade-TRAÇO $=44$ (Percentil 25 a 75), categorizada como Ansiedade Moderada de acordo com o IDATE, não houve associação positiva entre a situação da gestante no mercado de trabalho e escore de Ansiedade Traço (Tabela 1). 
No que se refere à situação conjugal, observou-se, neste estudo que as gestantes que referiram o estado civil como união estável ou consensual possuíam em sua maioria, tanto o Traço quando o Estado ansioso elevado, $23 \%$ e $19 \%$ respectivamente, ao contrário das que referiram ser casadas que possuem em sua maioria, tanto Traço quanto Estado ansioso moderado, 22\% e baixo 19\% (Tabela 2).

Independentemente da idade todas as gestantes apresentaram, em média, escore de ansiedade tanto traço como estado elevados (Tabela 3). A maioria 35 (66\%) encontra-se na faixa etária considerada adequada para a gestação (21 a 35 anos).

Ao testar as variáveis acima descritas com a ansiedade-Traço e a ansiedade-Estado, todas revelaram independência, exceto a variável raça/cor que mostrou diferença estatística significativa para a ansiedade-Estado ( $p=0,047)$, gestantes negras possuíam em média Estado ansioso elevado 56,25 ao contrário das gestantes brancas e pardas que possuíam escores em média de 49,83 e 47,06 respectivamente, categorizados com Estado ansioso moderado.

Tabela 1: Médias e Desvios padrões das variáveis maternas ansiedade e sociodemográficas segundo os três grupos de gestantes.

\begin{tabular}{|c|c|c|c|c|c|c|}
\hline \multirow[t]{2}{*}{ Variáveis maternas } & \multicolumn{2}{|c|}{$\begin{array}{l}\text { Gestante Adolescente } \\
\text { Até } 20 \text { anos }\end{array}$} & \multicolumn{2}{|c|}{$\begin{array}{c}\text { Gestante Jovem } \\
\text { Entre } 21 \text { e } 35 \text { anos }\end{array}$} & \multicolumn{2}{|c|}{$\begin{array}{c}\text { Gestante Tardia } \\
\text { A partir de } 35 \text { anos }\end{array}$} \\
\hline & $\mathrm{n}(\%)$ & $M \pm D P$ & $\mathrm{n}(\%)$ & $M \pm D P$ & $\mathrm{n}(\%)$ & $M \pm D P$ \\
\hline \multicolumn{7}{|l|}{ Ansiedade } \\
\hline Tracco & $14(26)$ & $46,5 \pm 11,73$ & $35(66)$ & $47,68 \pm 9,30$ & $4(8)$ & $44,5 \pm 15,26$ \\
\hline $\begin{array}{l}\text { Estado } \\
\text { Idade }\end{array}$ & $14(26)$ & $\begin{array}{l}46,42 \pm 10,52 \\
18 \pm 1,96\end{array}$ & $35(66)$ & $\begin{array}{l}50,68 \pm 10,57 \\
27,8 \pm 3,66\end{array}$ & $4(8)$ & $\begin{array}{l}44,25 \pm 18,83 \\
39 \pm 3,46\end{array}$ \\
\hline Escolaridade (anos) & & $12,14 \pm 3,20$ & & $12,42 \pm 2,93$ & & $14 \pm 2$ \\
\hline Ensino Fundamental & $6(11)$ & & $12(23)$ & & $0(0)$ & \\
\hline Ensino Médio & $5(9)$ & & 16(30) & & $3(6)$ & \\
\hline Ensino Superior & $3(6)$ & & $7(13)$ & & $1(2)$ & \\
\hline Renda Familiar & & $1,85 \pm 0,94$ & & $2,11 \pm 0,96$ & & $1,75 \pm 0,95$ \\
\hline Menor que 1 salário & $6(11)$ & & $8(15)$ & & $2(4)$ & \\
\hline Entre 1 e 2 salários & $5(9)$ & & $19(36)$ & & $0(0)$ & \\
\hline Entre 2 e 3 salários & $2(4)$ & & $5(9)$ & & $1(2)$ & \\
\hline Entre 3 e 4 salários & 1(2) & & 1(2) & & 1(2) & \\
\hline Mais de 4 salários & $0(0)$ & & 2(4) & & $0(0)$ & \\
\hline$N^{\circ}$ de Gestações & & $1,21 \pm 0,42$ & & $2,05 \pm 1,05$ & & $2,50 \pm 0,57$ \\
\hline Primigesta & $11(20)$ & & $13(25)$ & & $0(0)$ & \\
\hline Gesta II & $3(6)$ & & 11(20) & & $2(4)$ & \\
\hline Gesta III & $0(0)$ & & $8(15)$ & & $2(4)$ & \\
\hline Gesta IV & $0(0)$ & & $2(4)$ & & $0(0)$ & \\
\hline Gesta V & $0(0)$ & & $1(2)$ & & $0(0)$ & \\
\hline $\begin{array}{l}\mathrm{N}^{\circ} \text { de moradores no } \\
\text { domicílio } \\
\text { Raça/Cor }\end{array}$ & & $4 \pm 1,88$ & & $3,37 \pm 1,26$ & & $3,25 \pm 0,5$ \\
\hline Brancas & $2(4)$ & & $8(15)$ & & $2(4)$ & \\
\hline Pardas & $9(17)$ & & $24(42)$ & & 1(2) & \\
\hline Negras & $3(6)$ & & $4(8)$ & & $1(2)$ & \\
\hline
\end{tabular}


Tabela 2: Percentual dos escores no IDATE-T e IDATE-E de acordo com o estado civil das gestantes do estudo.

\begin{tabular}{llcllll}
\hline & & \multicolumn{3}{c}{ Ansiedade-TRAÇO } & \multicolumn{3}{c}{ Ansiedade-ESTADO } \\
\hline Variáveis Maternas & $\begin{array}{l}\text { Baixa } \\
\mathrm{n}(\%)\end{array}$ & $\begin{array}{l}\text { Moderada } \\
\mathrm{n}(\%)\end{array}$ & $\begin{array}{l}\text { Elevada } \\
\mathrm{n}(\%)\end{array}$ & $\begin{array}{l}\text { Baixa } \\
\mathrm{n}(\%)\end{array}$ & $\begin{array}{l}\text { Moderada } \\
\mathrm{n}(\%)\end{array}$ & $\begin{array}{l}\text { Elevada } \\
\mathrm{n}(\%)\end{array}$ \\
Estado Civil n(\%) & & & & & & \\
Casada 21(40) & $3(6)$ & $11(21)^{\neq}$ & $7(13)$ & $10(19)^{\mp}$ & $7(13)$ & $4(8)$ \\
Solteira 16(30) & $1(2)$ & $5(9)$ & $10(19)$ & $2(4)$ & $7(13)$ & $7(13)$ \\
Separada 01(2) & - & - & $1(2)$ & - & $1(2)$ & - \\
União Consensual 15(28) & - & $3(6)$ & $12(22)$ & - & $5(9)$ & $10(19)$ \\
\hline
\end{tabular}

${ }^{*} \mathrm{p}<0,05$ entre os grupos teste ANOVA e Qui-Quadrado de Pearson

Tabela 1: Percentual dos escores no IDATE-T e IDATE-E de acordo com a raça/cor das gestantes do estudo.

\begin{tabular}{lllllll}
\hline & \multicolumn{3}{c}{ Ansiedade-TRAÇO } & \multicolumn{3}{c}{ Ansiedade-ESTADO } \\
\hline Raça/Cor $\mathrm{n}(\%)$ & Baixa & Moderada & Elevada & Baixa & Moderada & Elevada \\
& $\mathrm{n}(\%)$ & $\mathrm{n}(\%)$ & $\mathrm{n}(\%)$ & $\mathrm{n}(\%)$ & $\mathrm{n}(\%)$ & $\mathrm{n}(\%)$ \\
Branca 12(23) & $1(2)$ & $4(8)$ & $7(13)$ & $4(8)$ & $2(4)$ & $6(11)$ \\
Parda 33(62) & $3(6)$ & $14(26)$ & $16(30)$ & $7(13)$ & $16(30)$ & $10(19)$ \\
Negra 8 (15) & - & $1(2)$ & $7(13)^{\neq}$ & $1(2)$ & $2(4)$ & $5(9)^{\mp}$ \\
\hline
\end{tabular}

${ }^{*} \mathrm{p}<0,05$ entre os grupos teste ANOVA

\section{DISCUSSÃO}

Quanto à idade no início da gestação, tem-se 0 número de gestação precoce $14(26,8 \%)$ está acima da média estadual e nacional que são $21,19 \%$ e $19,25 \%$, respectivamente. ${ }^{7}$ Pesquisa realizada na região têm identificado prevalência de gestações não planejadas, evidenciando a fragilidade na assistência ao planejamento reprodutivo. ${ }^{8}$ Já as gestações tardias têm crescido ao longo dos anos. 0 número de mulheres que tiveram o primeiro filho após os 30 anos passou de 27,5\% em 2000 para $31,3 \%$ em 2010, um aumento de $14 \%{ }^{9}$

A postergação da gestação tornase mais frequente por decisão da própria mulher, devido ao desejo de sua independência, ao casamento adiado, carreira profissional sem intercorrências e ao melhor nível socioeconômico. A mulher, coloca em segundo plano a gestação para se dedicar à carreira profissional, adquirir estabilidade financeira, almejando não terem a gravidez afetada por questões financeiras e, o parceiro ideal. ${ }^{10-12}$ Enquanto que na adolescência acontece a gravidez, na maioria das vezes, indesejada, com um parceiro igualmente jovem, levando à evasão escolar e às uniões consensuais. ${ }^{13}$

Como o Brasil ainda é um país em desenvolvimento, a gestação tardia tem se tornado muito frequente, o percentual de gestantes com idade igual ou superior a 35 anos tem aumentado, conforme descrito anteriormente. Todavia a transição demográfica e epidemiologia deve ser 


\section{JOURNALOF \\ NURSING \\ ANO HEALTH}

ISSN 2236 - 1987

considerada, em especial ao que tange a saúde reprodutiva da mulher. ${ }^{14}$ Preocupações referentes a idade materna tem sido cada vez maior. ${ }^{10}$ Sabe-se que mulheres adolescentes e em idade avançada, são consideradas de maior risco para resultados perinatais adversos, tais como: anormalidades cromossômicas, aumento das taxas de abortamento, pré-eclâmpsia e mortalidade materna. ${ }^{12}$ A maioria dos autores esclarece que gestantes adolescentes e tardias estão suscetíveis à resultados perinatais adversos e à morbimortalidade materna. Mulheres com gestação tardia apresentam risco similar às adolescentes em alguns aspectos, e mais elevado em outras situações como o abortamento espontâneo, gravidez ectópica, anormalidades cromossômicas e malformações congênitas. ${ }^{15}$

Apesar da tendência do aumento na incidência da gravidez nos extremos da vida reprodutiva, antes dos 20 e após os 35 anos de idade, seja uma realidade, ${ }^{16}$ os achados do presente estudo permitem inferir a incidência majoritária da gravidez na faixa etária considerada ideal para o seu desenvolvimento (Tabela 2), a qual varia entre 20 e 35 anos.

A média de filhos identificada é menor que a nacional ${ }^{9}$ (Tabela 2) as mulheres têm postergado a gestação e possuem, em média, 1,9 filhos. 0 acumulo de papéis vivenciados pelas mulheres também é um fator que pode estar contribuído para a diminuição da taxa de natalidade.

Os achados quanto ao estado civil (Tabela 3) estão em consonância com o Departamento de Informática do
Sistema Único de Saúde (DATASUS), no ano de 2014 no estado do MT, houve predomínio de $70,9 \%$ de mulheres em união estável e casadas. 0 estado civil é influente na conduta emocional e psíquica da grávida, as mulheres casadas dispõem de maior apoio e, por isso, menos ansiedade e até hostilidade durante a gravidez, quando comparadas com as solteiras, divorciadas ou viúvas. ${ }^{17}$ Isto também pode ser identificado neste estudo pois as gestantes casadas apresentaram o escore tanto Traço quanto Estado ansioso moderado enquanto que as gestantes, que referiram o estado civil como união estável ou consensual apresentaram tanto o Traço quando o Estado ansioso elevado (Tabela 2).

Esta constatação leva-nos afirmar que existe dependência entre a variável estado civil, tanto para a ansiedade-traço quanto para a ansiedade-estado, o que equivale a afirmar que ser casada tem efeito significativo na ansiedade, ou seja, as variáveis são dependentes.

Segundo o Instituto Brasileiro de Geografia e Estatística (IBGE) a maioria das brasileiras possui entre 11 e 14 anos de estudo, ${ }^{18}$ o mesmo pode ser identificado entre as gestantes deste estudo. Identificou-se ainda que a minoria das mulheres concluiu o ensino superior, resultado já esperado uma vez que se trata de um país subdesenvolvido. Todavia, as gestantes, entrevistadas, não apresentam baixa escolaridade visto que $43 \quad(81 \%)$ das gestantes apresentaram ensino fundamental e médio completos.

Contudo as gestantes jovens são as que possuem maior percentual de 


\section{JOURNALOF \\ NURSING \\ ANO HEALTH \\ ISSN 2236 - 1987}

ensino superior e a maior média de renda mensal. A escolaridade afeta o nível de conhecimento e a acessibilidade destas gestantes aos serviços de saúde. A inadequação da assistência de pré-natal está associada à baixa escolaridade. Gestantes com ensino superior completo e com acesso a serviços privados de saúde conseguiram realizar todas as consultas de pré-natal preconizadas pelo Ministério da Saúde. ${ }^{19} \mathrm{~A}$ vulnerabilidade das mulheres de menor escolaridade está associada ao menor acesso delas a uma rede de suporte familiar e/ou social tais como acesso a serviços de atenção à saúde. ${ }^{20} \mathrm{~A}$ educação, infundida na escolaridade, aumenta as possibilidades e escolhas de vida, bem como aquisição de novos conhecimentos que motivam atitudes e comportamentos saudáveis, afetando dessa forma diretamente a saúde dos indivíduos em consonância com os achados deste estudo. ${ }^{21}$

Quanto à ocupação há uma desvalorização da mulher "do Lar" em decorrência da valorização da "mulher profissional”, essa desvalorização pode contribuir para uma vivência desfavorável da gestação onde na maioria das vezes, essas mulheres deixam de se cuidar, física e psicologicamente, para cuidar da casa, companheiro e dos demais filhos. As dificuldades enfrentadas pelas mulheres no mercado de trabalho referem-se à qualidade dos empregos por elas obtidos, onde, encontra-se em maior proporção, em postos de trabalho suscetíveis, representados pelo assalariamento sem carteira assinada, trabalho doméstico e autônomas, desprotegendo-as assim de qualquer regulamentação que lhes garanta direitos sociais como, carteira de trabalho assinada, licença maternidade, entre outros. ${ }^{20}$

No contexto gestacional, deve-se pensar na possibilidade de a gestante economicamente inativa "do lar" ficar preocupada com o futuro, no que diz respeito à falta de emprego, de renda e do aumento dos gastos da família, o que pode acarretar motivo de ansiedade.

É importante refletir sobre a associação da renda mensal familiar com a ocupação, durante a gravidez, a ocupação como fonte de renda pode proporcionar uma sensação de tranquilidade à gestante, uma vez que a presença do salário mensal diminui as inquietações e a ansiedade referentes aos gastos advindos deste período com a preparação para o nascimento do filho. Ao tratamento estatístico, esta variável não apresentou efeito significativo na ansiedade.

0 fato de haver ansiedade elevada nas gestantes nas diferentes faixas etárias (Tabela 1), corrobora com os achados na literatura que afirmam que o estado gestacional por si só já causa ansiedade nas mulheres. ${ }^{22-23}$

A análise da paridade com a escolaridade corrobora com a constatação que os altos índices de natalidade estão relativamente mais presentes em gestantes com baixa escolaridade, enquanto os dados mostram uma diminuição da taxa de fecundidade nas classes sociais mais instruídas. $^{24}$ Portanto a taxa de natalidade é ascendente nas classes socioeconômico-culturais com menores índices de escolaridade, são 
justamente as classes que se supõe menor acesso às informações.

Os achados quanto ao número de moradores conferem com os registros de que a média de moradores em cada domicílio brasileiro, em todas as regiões, é de no mínimo três a quatro pessoas, conforme o Censo 2010.9

A população negra em geral, e principalmente as mulheres negras, recebem atendimento de saúde mais precário que outros grupos no Brasil.2526 Fatores históricos, econômicos e sociais fazem com que este seja um dos grupos populacionais mais vulneráveis na sociedade nacional atualmente. ${ }^{27}$ Para a gestante negra, estar grávida significa uma sobreposição de conflitos, pois ser mulher, gestante e negra, a deixa mais vulnerável, pois ela tem que lidar com diferentes formas de rejeições, preconceitos e a discriminação da sociedade. ${ }^{28}$

\section{CONCLUSÃO}

Houve predominância de gestantes jovens, pardas, casadas, com ensino médio, do lar, economicamente ativas, de baixa renda. Embora as gestantes economicamente inativas terem apresentado Ansiedade-Traço Elevada, enquanto as economicamente ativas, Ansiedade-Traço Moderada, não houve correlação positiva, entre a situação da gestante no mercado de trabalho e o escore de AnsiedadeTraço.

Constatou-se que a variável estado civil casada apresentou diminuição na ansiedade, indicando dependência entre a variável estado civil, tanto para a ansiedade-Traço quanto para a ansiedade-Estado. Ser casada, implica menores níveis ansiogênicos.

A morbidade ansiosa foi comprovada neste estudo com gestantes, assim sugere-se que pesquisas e intervenções como: rodas de conversas, musicoterapia, orientações quanto às práticas de atividades físicas, dentre outras, sejam realizadas a fim de minimizar os efeitos adversos que a ansiedade pode causar no binômio e promover a saúde, principalmente em gestantes negras e solteiras pois, são as que se encontram em maior vulnerabilidade e mais suscetíveis ao aumento da ansiedade gestacional.

A limitação do estudo diz respeito à unidimensionalidade do IDATE, apesar do Inventário de Ansiedade ser considerado instrumento padrão na mensuração dos níveis de ansiedade. Reconhece-se a importância de estudos que analisem os aspectos afetivos-emocionais da ansiedade através de uma avaliação multidimensional.

\section{REFERÊNCIAS}

1 Conde A, Figueiredo B. Preocupações de mães e pais, na gravidez, parto e pós-parto. Anál psicol [Internet]. 2007 [acesso em 2018 set 26];25(3):381-98. Disponível em: http://www.scielo.mec.pt/pdf/aps/v 25n3/v25n3a06.pdf

2 Piccinini CA, Gomes AG, De Nardi T, Lopes RS. Gestação e a constituição da maternidade. Psicol estud [Internet]. 2008 [acesso em 2018 set 26];13(1):63$72 . \quad$ Disponível em: http://www.scielo.br/pdf/pe/v13n1/ v13n1a07.pdf 


\section{JOURNALOF \\ NURSING \\ ANO HEALTH}

ISSN 2236 - 1987

[acesso em 2018 set 26];7(1):58-66. Disponível em: https://periodicos.ufpel.edu.br/ojs2/ index.php/enfermagem/article/view/ 8951

9 Instituto Brasileiro de Geografia e Estatística (IBGE). Características da população e dos domicílios: resultados do universo [Internet]. Rio de Janeiro: IBGE; 2010 [acesso em 2018 abr 20]. Disponível em: http://ibge.gov.br/cidadesat/painel/ populacao.php?lang=\&codmun=510665 \&search=mato-grosso | pontal-doaraguaia | infograficos:-evolucaopopulacional-e-piramide-etaria.

10 Cohen WR. Does maternal age affect pregnancy outcome?. BJOG [Internet]. 2014 [cited 2018 Sept 26];121(3):252$4 . \quad$ Available from: https: / /obgyn.onlinelibrary.wiley.com /doi/epdf/10.1111/1471-0528.12563

11 Bezerra AC, de Mesquita JD, Brito $M D$, dos Santos RB, Teixeira FV. Desafios enfrentados por mulheres primigestas em idade avançada. Rev bras cienc saude [Internet]. 2016 [acesso em 2018 set 26];19(2):163-8. Disponível

em: http://www.periodicos.ufpb.br/ojs/in dex.php/rbcs/article/view/24335/150 65

12 Moura BLA, Alencar GP, Silva ZP, Almeida MF. Internações por complicações obstétricas na gestação e desfechos maternos e perinatais, em uma coorte de gestantes no Sistema Único de Saúde no Município de São Paulo, Brasil. Cad saúde pública (online) [Internet]. 2018 [acesso em 2018 set 26];34(1):1-12. Disponível em: http://www.scielo.br/pdf/csp/v34n1 /1678-4464-csp-34-01-e00188016.pdf 


\section{ISSN 2236 - 1987}

13 Vieira EM, Bousquat A, Barros CRS, Alves, MCGP. Gravidez na adolescência e transição para a vida adulta em jovens usuárias do SUS. Rev saude publica [Internet]. 2017 [acesso em 2018 set 26];51(25):1-10. Disponível em:

http://www.scielo.br/pdf/rsp/v51/pt _0034-8910-rsp-S1518-

87872016050006528.pdf

14 Pereira QLC, Silva CB, Siqueira HC. Processo de viver de mulheres climatérias usuárias do sistema único de saúde. Ciênc cuid saúde [Internet]. 2008 [acesso em 2018 set 26];7(2):224 $33 . \quad$ Disponível em: http://www.periodicos.uem.br/ojs/in dex.php/CiencCuidSaude/article/view /5006/3245

15 Bacelar EB, Costa MCO, Gama SGN, Amaral MTR, Almeida AHV. Factors associated with specific hypertensive gestation syndrome (SHGS) in postpartum adolescent and young adult mothers in the northeast of Brazil: a multiple analysis of hierarchical models. Rev bras saúde mater infant (online) [Internet]. 2017 [cited 2018 Sept 26];17(4):673-81. Available from: http://www.scielo.br/pdf/rbsmi/v17n 4/1519-3829-rbsmi-17-04-0673.pdf

16 Rodrigues APA, Santos LBB, Castela MPS, França AMB, Pimpão FD. Análise da prematuridade nos extremos da idade reprodutiva. Interfaces científicas [Internet]. 2015 [acesso em 2018 nov 08];3(3):65-78. Disponível em:

https://periodicos.set.edu.br/index.p $\mathrm{hp} /$ saude/article/view/2107/pdf_4

17 Silveira C, Ferreira MM. Autoconceito da grávida: fatores associados. Millenium [Internet]. 2016 [acesso em 2018 set 26];40:53-67. Disponível

em: https://revistas.rcaap.pt/millenium/a rticle/view/8219

18 Instituto Brasileiro de Geografia e Estatística (IBGE). Censo: resultados da amostra-características da população [Internet]. Rio de Janeiro: IBGE; 2010 [acesso em 2017 nov 17]. Disponível em:

https://ww2.ibge.gov.br/home/estati stica/populacao/condicaodevida/indic adoresminimos/suppme/default_educ acao.shtm

19 Viellas EF, Domingues RMSM, Dias MAB, Gama SGN, Filha MMT, Costa JV, et al. Prenatal care in Brazil. Cad saúde pública [Internet]. 2014 [cited 2018 Sept 26];30(Suppl1):85-100. Available from: http://www.scielo.br/pdf/csp/v30s1/ en_0102-311X-csp-30-s1-0085.pdf

20 Monteiro FR, BucciniGS, Venâncio $\mathrm{SI}$, Costa, Teresa HM. Influence of maternity leave on exclusive breastfeeding. J pediatr (Rio J) [Internet]. 2017 [cited 2018 Sept 26];93(5):475-81. Available: http://www.scielo.br/pdf/jped/v93n 5/0021-7557-jped-93-05-0475.pdf

21 Rocha AA, Cavalcanti TCFA. Depressão pós-parto: uma revisão sobre fatores de risco e de proteção. Psicol saúde doenças [Internet]. 2017 [acesso em 2018 set 26];18(3):828-45. Disponível em: http://www.redalyc.org/pdf/362/362 54714016.pdf

22 Silva MMJ, Leite EP, Nogueira DA, Clapis MJ. Ansiedade e depressão na gravidez: caracterização de gestantes que realizaram pré-natal em unidades 


\section{JOURNALOF \\ NURSING \\ ANO HEALTH}

ISSN 2236 - 1987

à saúde e as desigualdades raciais: uma proposta de intervenção. Saúde soc [Internet]. 2016 [acesso em 2018 set 26];25(3):689-702. Disponível em: http://www.scielo.br/pdf/sausoc/v25 n3/1984-0470-sausoc-25-03-00689.pdf

27 Fraga FA, de Carvalho Sanino GE. Saúde da mulher negra passos e descompassos: ações afirmativas na saúde, provável luz no fim do túnel? Rev ABPN [Internet]. 2015 [acesso em 2018 set 26];7(15):192-211. Disponível em:

http://www.abpnrevista.org.br/revist a/index.php/revistaabpn $1 /$ article/vie $\mathrm{w} / 121 / 118$

28 Costa DO, Souza FISD, Pedroso GC, Strufaldi MWL. Transtornos mentais na gravidez e condições do recémnascido: estudo longitudinal com gestantes assistidas na atenção básica. Ciênc saúde colet [Internet]. 2018 [acesso em 2018 nov 06];23:691-700. Disponível em: http: //www.scielo.br/pdf/csc/v23n3/ 1413-8123-csc-23-03-0691.pdf

Data de submissão: 12/05/2018

Data de aceite: 06/11/2018

Data de publicação: 12/11/2018

https://revistainterdisciplinar.uninova fapi.edu.br/index.php/revinter/articl e/view/1133

25 Carneiro R. 0 peso do corpo negro feminino no mercado da saúde: mulheres, profissionais e feministas em suas perspectivas. Mediações: revista de ciências sociais [Internet]. 2017 [acesso em 2018 set 26];22(2):394-424. Disponível em: http://www.uel.br/revistas/uel/inde x.php/mediacoes/article/view/23609 /pdf

26 Batista LE, Rattner D, Kalckmann S, Oliveira MCG. Humanização na atenção 